\title{
Basic and translational research in the European Respiratory Journal
}

\author{
Ramon Farré ${ }^{1,2,3}$, Pieter S. Hiemstra $\mathbb{1}^{4}$ and Anh Tuan Dinh-Xuan ${ }^{5}$
}

Affiliations: 'Unitat Biofísica i Bioenginyeria, Facultat de Medicina i Ciències de la Salut, Universitat de Barcelona, Barcelona, Spain. ${ }^{2} \mathrm{CIBER}$ de Enfermedades Respiratorias, Madrid, Spain. ${ }^{3}$ Institut Investigacions Biomediques August Pi Sunyer, Barcelona, Spain. ${ }^{4}$ Dept of Pulmonology, Leiden University Medical Center, Leiden, The Netherlands. ${ }^{5}$ Heart and Lung Dept, Cochin Hospital, Paris Descartes University, Paris, France.

Correspondence: Ramon Farré, Unitat Biofísica i Bioenginyeria, Facultat de Medicina i Ciències de la Salut, Casanova 143, 08036 Barcelona, Spain. E-mail: rfarređuub.edu

\section{@ERSpublications}

The proportion of papers on basic science published in the European Respiratory Journal has decreased in recent years, but those published have similar citation rates to papers dealing with clinical research http://ow.ly/2SW230jptQR

Cite this article as: Farré R, Hiemstra PS, Dinh-Xuan AT. Basic and translational research in the European Respiratory Journal. Eur Respir J 2018; 51: 1800377 [https://doi.org/10.1183/13993003.00377-2018].

The impressive advancements in health quality and life expectancy achieved in recent decades have been made possible by successful efforts in investigation and innovation. The scientific discoveries coming from basic research in a variety of disciplines such as biology, pharmacology, immunology, epidemiology, physics and engineering are the very first steps in the long journey leading to progress in healthcare. It is important to note that a considerable number of scientific breakthroughs resulting in relevant medical advances initially derived from research that was originally not focused on its final clinical application. Translation of such findings from basic research and preclinical testing in cellular and animal models is a next step in the development of new tools such as preventive and therapeutic drugs, medical devices for diagnosis and treatment, or new procedures. Finally, evaluating the application of these novel tools by means of large scale clinical research and trials, considering both effectiveness and cost, is mandatory before incorporating innovations into the daily routine of patient care.

Initial steps in biomedical research are frequently the result of research performed in groups not directly linked to clinical care; therefore, the translational potential of this research needs to be identified by the researchers themselves as well as by those involved in ultimately bringing these results to the clinic. Therefore, it is essential for healthcare professionals to be exposed to these results to take full advantage of the advancements in basic/translational science. To this end, medical congresses and expert symposia are well established platforms for dissemination. However, this process is hampered by the number of healthcare professionals potentially interested in getting recent updates on breakthroughs in basic/ translational science. Medical journals have been another means of communicating progress in healthcare since their birth in the 17 th century [1], and today thanks to digital publication they can reach readers worldwide in real time.

Although publications in such medical journals are at the core of dissemination of advances in healthcare, these journals are not comprehensive tools since they have different profiles and very specific readerships. On one side of the spectrum there are journals focused on basic/translational research that mainly target non-clinician scientists. These journals may cover a wide range of topics in biomedicine (e.g. Cell, FASEB Journal and Science Translational Medicine) or may be devoted to a specialty readership, for instance respiratory medicine (e.g. American Journal of Respiratory Cell and Molecular Biology and American 
Journal of Physiology - Lung Cellular and Molecular Physiology). On the other side of the spectrum there are journals that almost exclusively target a clinical readership, again focused on general medicine (e.g. The New England Journal of Medicine and JAMA) or on a specialty (e.g. Lancet Respiratory Medicine). In between these categories there are medical journals, such as the European Respiratory Journal (ERJ), that publish both basic/translational and clinical research in different proportions to provide their readers with a wide perspective on how the field is evolving [2]. However, given the skyrocketing number of studies generated in the past decade (figure 1), it is not easy for the editors of this type of journal to allocate both basic/translational and clinical papers within the limited space available in each journal issue.

In fact, there has been a continuous decrease in basic/translational research articles in general and specialty medical journals in recent years, as reported by STEINBERG et al. [3]. This interesting analysis investigated the proportion of basic/translational science papers in the highest impact journals of eight medical specialties and reported that there was a considerable reduction, of $40-60 \%$, in basic/translational science papers published in the period from 1994 to 2013, irrespective of the medical specialty. Remarkably, the steepest reduction was in the past decade. In line with this, the proportion of basic/ translational science publications in the ERJ has experienced a similar trend. Indeed, a detailed analysis of all citable publications (i.e. editorials, reviews, originals, research letters and correspondence) in the ERJ shows a remarkable reduction in publications dealing with basic/translational science in recent years, from a maximum of $12 \%$ in 2009 to only $2 \%$ in 2014-2017 (figure 2a). Although these figures cannot be directly compared with the ones provided by STEINBERG et al. [3], since establishing a clear distinction between what is considered basic/translational or clinical research depends on the criteria used [3], the proportion of basic/translational science publications in the ERJ seems to be clearly below those reported for different medical specialties including respiratory medicine [3].

However, it is interesting to note that the basic/translational research publications in the ERJ received a similar number of citations as the total number of publications (which in a huge proportion deal with clinical research). This is illustrated in figure $2 \mathrm{~b}$, showing that during the period 2008-2014, the average number of citations per year per publication in the 2 years following its publication (the citations used to compute the impact factor) was 3.67. The figure also shows the values computed for the expanded period from the year following publication until 2016. Comparison of these two figures gives an approximate impression of the persistence of citations. As shown in figure $2 \mathrm{~b}$, the total ERJ publications have received quite a constant number of citations per year per paper, regardless of the duration of the period taken for computing this citation index. Interestingly, figure $2 \mathrm{c}$ displays the same citation indices for the ERJ papers dealing with basic/translational science and shows that the average number of citations was 3.17 per year per paper over the whole period, a value quite similar ( $\sim 15 \%$ lower $)$ to the one found for all publications (3.67). The fluctuation observed in recent years is probably caused by the reduced number of basic/ translational science papers published in those years (figure 2a). Interestingly, the persistence of citations over the period longer than 2 years was also very high for basic/translational science papers. Hence, figures $2 \mathrm{~b}$ and $2 \mathrm{c}$ indicate that basic/translational research papers published in $E R J$, although relatively small in number, had similar citation rates as the ones describing clinical research.

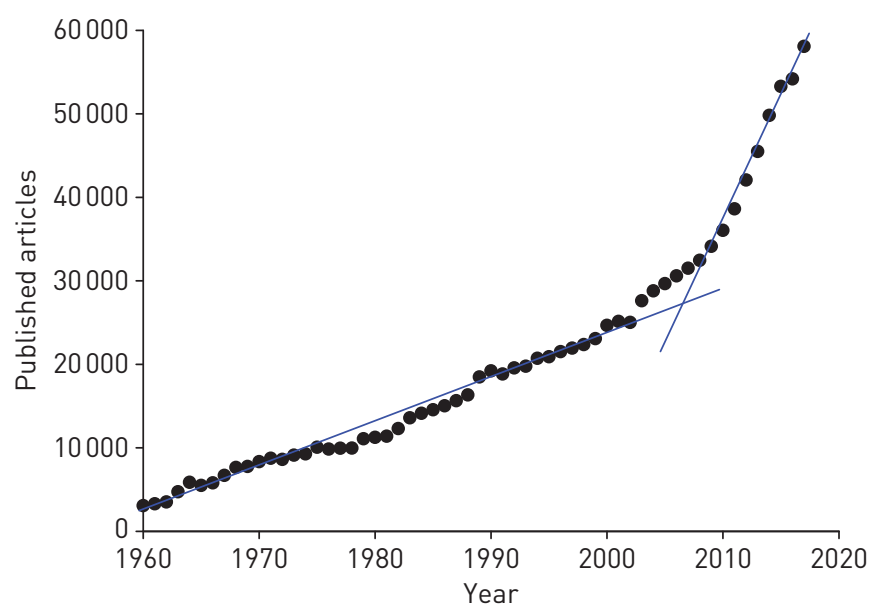

FIGURE 1 Number of papers published per year based on a PubMed search using the terms: "Respiratory OR Lung" (www.ncbi.nlm.nih.gov/pubmed/?term=respiratory+OR+lung). A first phase with constant growth rate extends from 1960 to 2000 . The past decade (2008-2017) is characterised by an almost six-fold greater constant growth rate. 

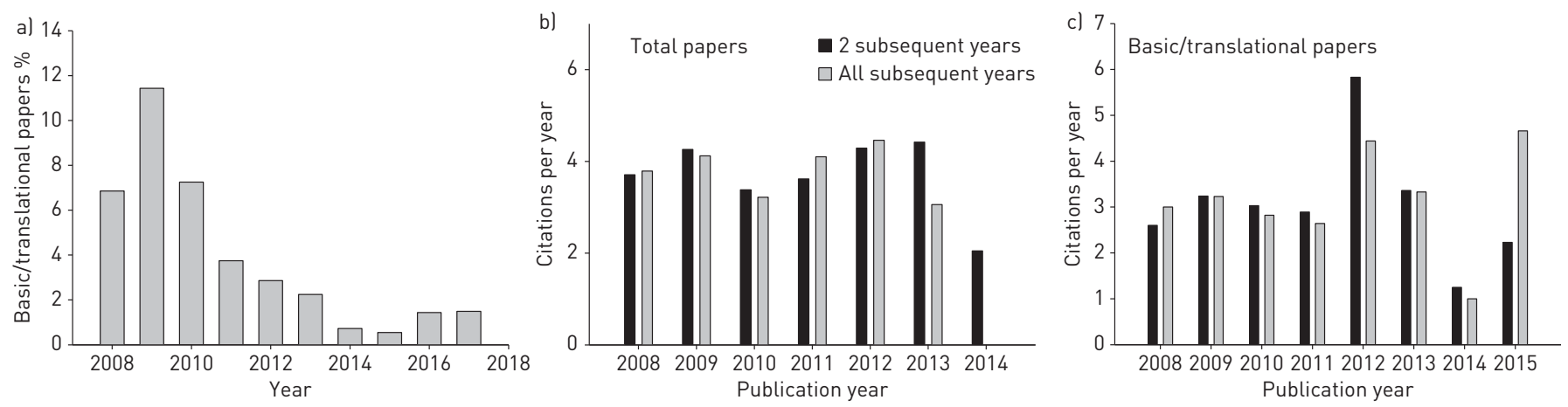

FIGURE 2 a) Percentage of publications dealing with basic/translational science in the European Respiratory Journal (ERJ). Mean number of citations per year b) for all papers and c) for basic/translational science papers published by the ERJ. For each year the figure shows the mean citations per year per publication for the 2 years after the year of publication (2 subsequent years) and for the expanded period from the year following publication until 2016 (all subsequent years).

Achieving a good balance between quantity and quality is always a problem in any activity and in particular in medical journal editorship. Whereas journals that are business-driven can be very selective, non-profit journals, such the ERJ, which are aimed at serving the society and the professional community, should ensure the highest levels of quality and facilitate widespread scientific communication simultaneously. The data in figure 2 indicate that the ERJ has recently published a relatively low number of basic/translational science papers as compared with other medical journals [3]. This lower number could either be explained by a low number of submissions and/or a higher rate of rejections of basic/ translational science as compared with clinical papers. Both explanations actually hold true, as the ERJ is mostly viewed as a clinical journal that may be considered less attractive for researchers involved in basic/ translational science who therefore do not submit their manuscripts (or at least their best ones) to it. However, the quality and visibility of the basic/translational science published by ERJ, measured by the number of citations, was quite high: only $\sim 15 \%$ lower than the citations for clinically focused papers, which compares very well with data indicating that in the cardiovascular field the citation of papers in clinical journals was $34 \%$ higher than in basic science journals [4]. However, it is important to stress that regardless of publication metrics, basic/translational science is a fundamental component for the education and continuing professional development of physicians $[5,6]$. With the arrival of targeted therapies and personalised medicine based on multidisciplinary patient characteristics, this is even more important in today's medicine. Furthermore, as highlighted in the ERS statement on optimizing experimental research in respiratory diseases published in this issue [7], visibility of basic/translational science in more clinically oriented journals is essential to raise interest and stress the importance of fundamental science. Importantly, including both basic/translational and clinical science in medical journals and thus exposing a clinical readership to such scientific advances is essential to ensure an effective translation of research progress from the bench to the bedside for improving patient care. Therefore, it is important for high quality care of patients with respiratory diseases that basic scientists submit their best work to the ERJ explaining the translational potential of their research.

Conflict of interest: R. Farré reports grants from Antadir (contract between Antadir and University of Barcelona for bench testing CPAP devices) and ResMed (contract between ResMed and University of Barcelona for bench testing CPAP devices), outside the submitted work. P.S. Hiemstra reports grants from Boehringer Ingelheim (research grant to department) and Galapagos NV (research grant to department), outside the submitted work.

\section{References}

1 Colman E. The first English medical journal: Medicina Curiosa. Lancet 1999; 354: 324-326.

2 Farré R, Dinh-Xuan AT. Translational research in respiratory medicine. Eur Respir J 2007; 30: 1041-1042.

3 Steinberg BE, Goldenberg NM, Fairn GD, et al. Is basic science disappearing from medicine? The decline of biomedical research in the medical literature. FASEB J 2016; 30: 515-518.

4 Opthof T. Differences in citation frequency of clinical and basic science papers in cardiovascular research. Med Biol Eng Comput 2011; 49: 613-621.

5 Finnerty EP, Chauvin S, Bonaminio G, et al. Flexner revisited: the role and value of the basic sciences in medical education. Acad Med 2010; 85: 349-355.

6 Woods NN, Brooks LR, Norman GR. The value of basic science in clinical diagnosis: creating coherence among signs and symptoms. Med Educ 2005; 39: 107-112.

7 Bonniaud P, Fabre A, Frossard N, et al. Optimising experimental research in respiratory diseases: an ERS statement. Eur Respir J 2018; in press [https://doi.org/10.1183/13993003.02133-2017]. 